\title{
Piles of profiles help identify vulnerabilities
}

Central nervous system (CNS) tumours are the most common solid tumours in children. Although these tumours are heterogeneous, many have a very poor prognosis as they are aggressive and have few treatment options. Lin et al. and Lambo et al. report in-depth therapeutic screening and genomic profiling, respectively, of two types of aggressive paediatric CNS tumours, and both groups identify vulnerabilities of these tumours that might be exploited therapeutically.

Diffuse midline gliomas (DMGs), including diffuse intrinsic pontine glioma (DIPG) and other gliomas that also carry a recurrent mutation in histone $\mathrm{H} 3$, have a universally lethal prognosis. To facilitate preclinical research and address the urgent need for new therapies in DMGs, Lin et al. conducted high-throughput screens of libraries containing 2,706 unique drugs in six DIPG patient-derived cell culture models; they also examined CNS penetration of these agents as this will be essential for their clinical translation. The proteasome inhibitor marizomib and the pan-histone deacetylase (HDAC) inhibitor panobinostat emerged as the most promising candidates.

As successful therapy is likely to require combinations, marizomib and panobinostat were analysed in combination with a large number of drugs from one library, and 45 selected agents (identified in the screen or currently in clinical trials for DIPG) were further tested against each other. Overall, 9,195 different drug combinations were evaluated. Several promising combinations were identified, and the authors have made this screening data publicly available. Panobinostat is already in phase I clinical trials for DIPG and had synergy with marizomib and other agents, so the authors further investigated these combinations. In an orthotopic patient-derived xenograft (PDX) model, only the combination with marizomib increased animal survival. Similar results were observed in orthotopic PDX models of other DMGs (spinal cord and thalamic gliomas).

both groups
identify
vulnerabilities
of these
tumours
that might
be exploited
therapeutically

Transcriptional profiling of cells treated with marizomib and panobinostat in combination identified upregulation of the unfolded protein response and downregulation of several metabolic pathways. Analysis of metabolites in DMG cultures indicated that combination therapy substantially reduced $\mathrm{NAD}^{+}$ levels, and supplementation with a $\mathrm{NAD}^{+}$precursor blocked cytotoxicity, indicating that this metabolic dysfunction is key for the efficacy of marizomib and panobinostat.

Our understanding of the molecular landscape of another aggressive paediatric CNS tumour type, embryonal tumours with multi-layered rosettes (ETMRs), is limited, so Lambo et al. profiled primary ETMRs and matched relapse samples in more detail. Amplification of a chromosome 19 microRNA (miRNA) cluster (C19MC) is known to occur in the majority of ETMRs; transcriptional profiling indicated that ETMRs without C19MC amplification often also had upregulation of C19MC (albeit at a lower level) as well as of the miR17-92 cluster. Genomic profiling showed that ETMRs have very few recurrently mutated genes, but miRNAs emerged as key: different mutations in C19MC, amplification of the miR-17-92 cluster and both germline and somatic mutations that disrupt the function of the miRNA-processing enzyme DICER1 were all observed. The authors suggest that amplified miRNAs might disrupt the miRNA processing machinery in a manner similar to



mutated DICER1; as such, these tumours seem molecularly similar.

Severe chromosomal instability, copy-number aberrations and breakpoints indicative of chromothripsis were also observed in many ETMR samples. These structural alterations seem to occur early in tumorigenesis as there was little variation of these between primary and relapse samples. Furthermore, although somatic single-nucleotide variants increased in relapse samples, these were poorly conserved and were most likely driven by chemotherapy. Together, these data suggest that the structural alterations are likely ETMR drivers.

One mechanism associated with increased chromosomal instability is the formation of R-loops (singlestranded DNA loops that can form during transcription). The density of R-loops was increased around C19MC and other breakpoints in the genome of ETMRs, and staining of ETMR samples with an R-loop-specific antibody indicated that the tumours contained a large number of R-loops. Interestingly, DICER1 has been associated with R-loop formation, thereby linking these observations.

Finally, the authors investigated whether R-loops might be exploited therapeutically in ETMRs. They previously found that ETMRs are sensitive to inhibitors of DNA topoisomerase 1 (TOP1); one function of TOP1 is to resolve R-loops. Previous studies have also shown that poly(ADP-ribose) polymerase 1 (PARP1) inhibition synergizes with TOP1 inhibition. Inhibitors of TOP1 and PARP1 in combination induced more death of ETMR cells in culture than did monotherapies and increased the number of $\mathrm{R}$-loops in the cells; in vivo preclinical evaluation is needed to determine whether this might be a viable clinical strategy.

It is hoped that these profiling efforts will lead to improved therapy for these often devastating paediatric tumours.

Sarah Seton-Rogers

ORIGINAL ARTICLES Lambo, S. et al.

The molecular landscape of ETMR at diagnosis and relapse. Nature 576, 274-280 (2019) | Lin, G. L. et al. Therapeutic strategies for diffuse midline glioma from high-throughput combination drug screening. Sci. Transl Med. 11, eaaw0064 (2019) RELATED ARTICLE Jones, D. T. W. et al. Molecular characteristics and therapeutic vulnerabilities across paediatric solid tumours. Nat. Rev. Cancer 19, 420-438 (2019) 